\title{
At-a-Glance
}

\section{Emergency department surveillance of injuries and head injuries associated with baseball, football, soccer and ice hockey, children and youth, ages 5 to 18 years, 2004 to 2014}

\author{
S. McFaull, MSc; J. Subaskaran, MPH; B. Branchard; W. Thompson, MSc
}

Tweet this article

Team sports are a popular recreational activity for Canadian youth. Figure 1 provides an eleven-year snapshot (2004 to 2014) of the number and proportion (per 100 000) of all injuries, as well as the number of head injuries, for children and youth aged 5 to 18 years participating in any of four key team sports: baseball, football, soccer and ice hockey. Data collected from the Canadian Hospitals Injury Reporting and Prevention Program (CHIRPP), ${ }^{1}$ an injury and poisoning surveillance system managed by the Public Health Agency of Canada, were used to create the figure (tables available upon request). CHIRPP currently operates in 11 pediatric and 6 general hospitals across Canada using an online data-entry system. The system is dynamic and is updated daily with new cases/information. CHIRPP does not capture all injuries in Canada, only those presenting to the participating emergency departments. However, a number of studies have indicated that the patterns are representative of the Canadian experience in certain contexts. ${ }^{2,3}$ Any cases considered non-relevant or containing errors were removed for this analysis.

The average annual percent change (AAPC) in all injuries reported through CHIRPP was calculated (with $95 \%$ confidence intervals) for each sport based on methods described by the National Cancer Institute. ${ }^{4}$ Over the 11-year period, the proportion of all injuries (number of total injuries per 100000 CHIRPP cases) due to baseball remained stable. Injuries due to football remained stable overall, but between 2004 and 2008 the proportion of injuries due to football rose at about $7 \%$ (95\% CI: 3.1-11.0) per year whereas between 2008 and 2014 there was a decrease of $2.2 \%$ ( $95 \% \mathrm{CI}:-3.9--0.5$ ) per year. Injuries due to soccer were also stable overall, but did show a $1.9 \%$ (95\% CI: 0.6-3.2) increase between 2007 and 2014. Injuries due to ice hockey were relatively stable over the 11 -year period, but there was a rising trend of $7.7 \%$
(95\% CI: 5.9-9.6) per year between 2006 and 2011 .

Overall, baseball had the highest proportion of reported head injuries (relative to all injuries) at $35.0 \%(1854 / 5300)$, followed by ice hockey at $27.2 \%$ (11 423/ 42 029), football (16.3\%; 3635/22 264) and soccer at $15.9 \%(7326 / 46$ 102). Except for baseball, which remained relatively stable, football, soccer and ice hockey show a $42 \%-47 \%$ increase in the proportion of head injuries in 2014 compared to 2004.

The following limitations are noted: increases in injury reported may be fully or partially explained by increased participation in sport or reporting to emergency rooms and are not necessarily due to an inherent increase in the danger/risk of the sport. Increases in the proportion of head injuries over time may be either due to actual increases in reported proportions, increased reporting through CHIRPP or a decrease in the numbers of non-head injuries. 
FIGURE 1

All injuries, head injuries, ${ }^{a}$ and number per 100000 CHIRPP injuries ${ }^{\mathrm{b}}$ by sport, ages 5-18, 2004-2014
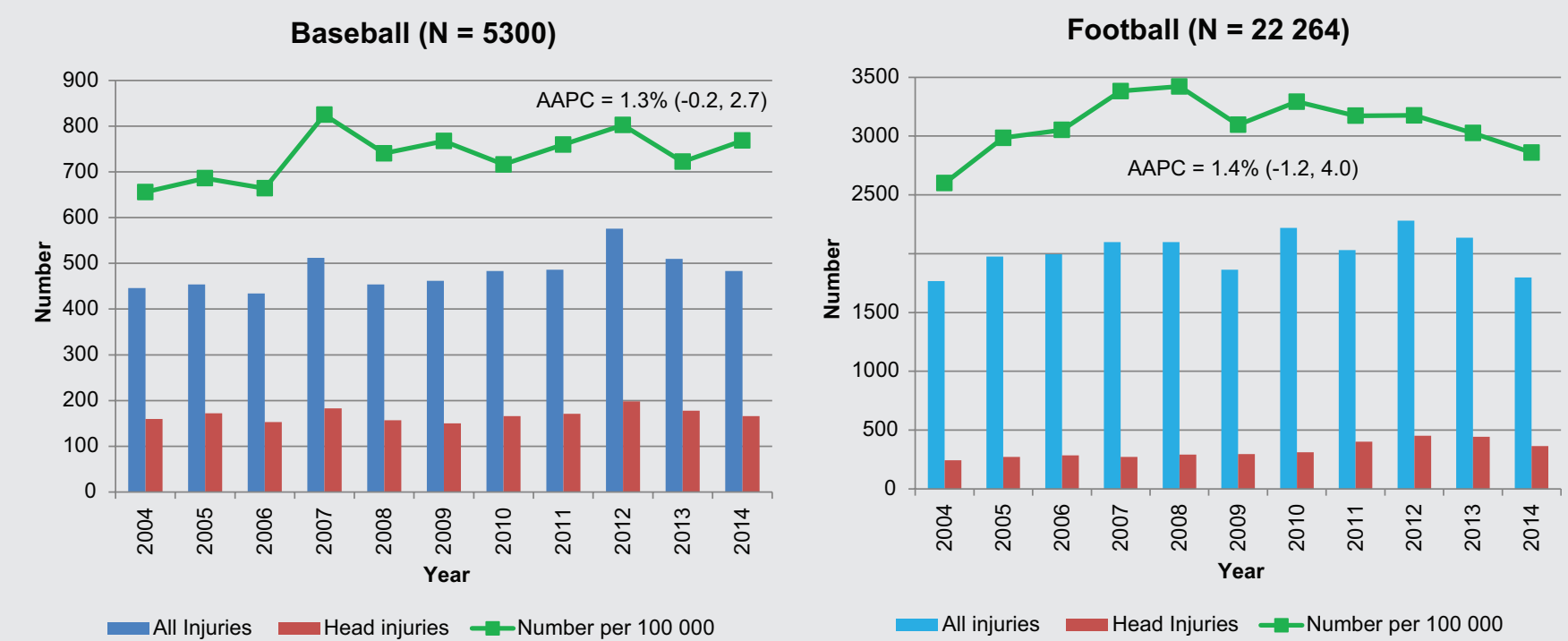

Soccer $(N=46$ 102)
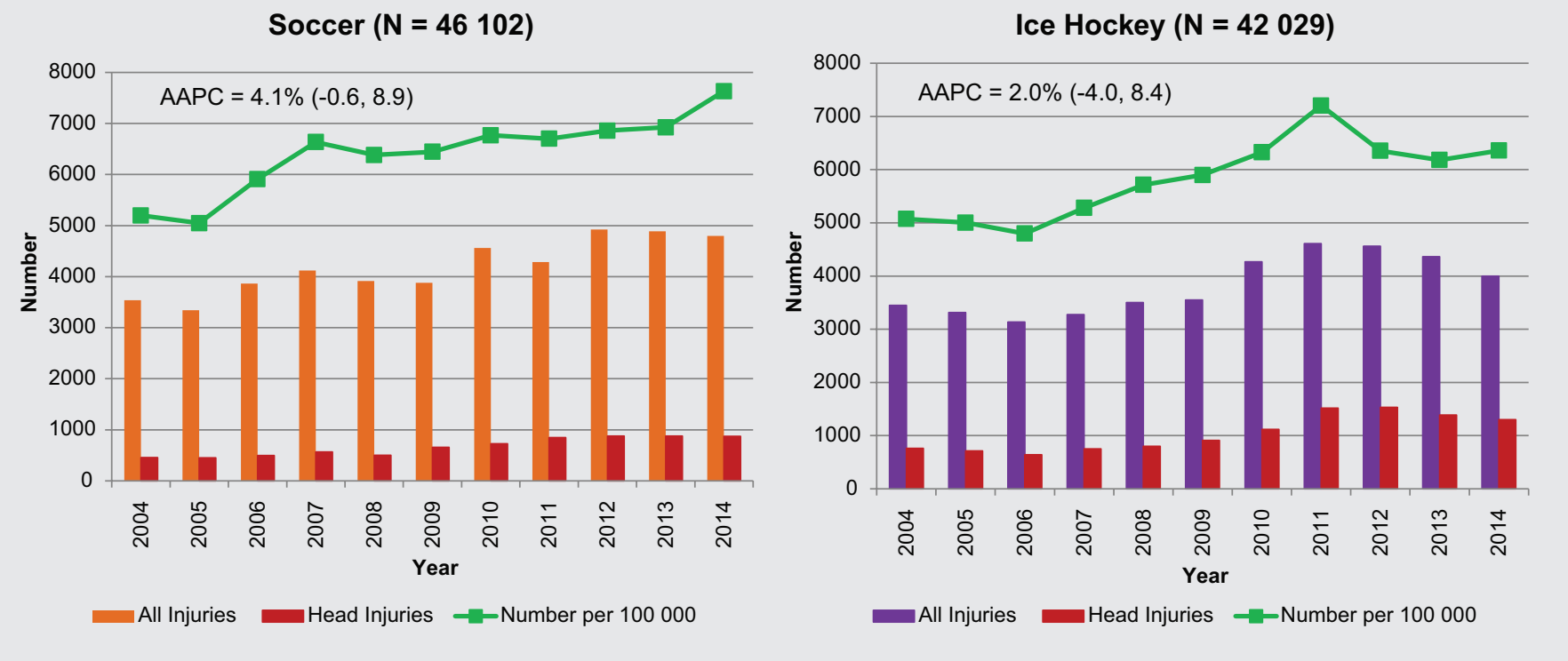

Abbreviations: AAPC, Average Annual Percent Change; CHIRPP, Canadian Hospitals Injury Reporting and Prevention Program.

Note: The AAPC in all injuries reported were calculated with $95 \%$ confidence intervals.

${ }^{a}$ Includes: skull and facial fractures, scalp and facial lacerations, dental injuries and brain injuries (minor closed head injury, concussion and intracranial injury).

${ }^{\mathrm{b}}$ Number of injuries per 100000 CHIRPP cases of all types for the given year, ages 5-18.

${ }^{\mathrm{C}}$ As of June 15, 2015. Counts for 2012-2014 are proportional estimates as information is still being entered into the CHIRPP system.

\section{References}

1. Mackenzie SG, Pless IB. CHIRPP: Canada's principal injury surveillance program. Inj Prev. 1999;5:208-13.

2. Pickett W, Brison RJ, Mackenzie SG, et al. Youth injury data in the Canadian Hospitals Injury Reporting and Prevention Program: do they represent the Canadian experience? Inj Prev. 2000;6:9-15.
3. Kang J, Hagel B, Emery CA, Senger T, Meeuwisse W. Assessing the representativeness of Canadian Hospitals Injury Reporting and Prevention Program (CHIRPP) sport and recreational injury data in Calgary, Canada. International Journal of Injury Control and Safety Promotion. 2013;20(1):19-26.
4. Average Annual Percent Change (AAPC) [Internet]. National Cancer Institute; [cited 2015 Sept 3]. Available from: http://surveillance cancer.gov/joinpoint/webhelp/Executing the_Joinpoint_Parameters/Statistical_Notes/ Statistics_Related_to_the_k-joinpoint_Model/ Average_Annual_Percent_Change.htm 